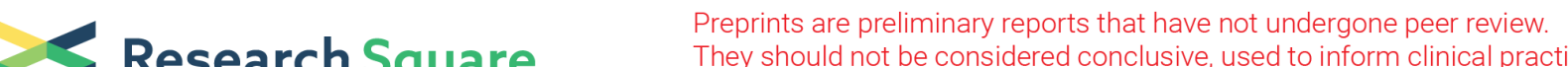 $\begin{array}{ll}\text { Research Square } & \text { They should not be considered conclusive, used to inform clinical practice, } \\ \text { or referenced by the media as validated information. }\end{array}$
}

\section{Discovery of a naturally-occurring allele of elF4E1.S in Nicotiana tabacum and development of a co- dominant marker}

Shifeng Lin ( $\square$ linshifeng1978@163.com )

Guizhou Academy of Tobacco Science

Ralph E. Dewey

North Carolina State University

Rengang Wang

Guizhou Academy of Tobacco Science

Jing Yu

Guizhou Academy of Tobacco Science

Mingjin Long

Guizhou Academy of Tobacco Science

Jie Zhang

Guizhou Academy of Tobacco Science

Shizhou Yu

Guizhou Academy of Tobacco Science

Jishun Zhang

Guizhou Academy of Tobacco Science

Zili Wang

Guizhou Academy of Tobacco Science

Xueliang Ren

Guizhou Academy of Tobacco Science

\section{Research article}

Keywords: Nicotiana tabacum, Potato virus Y, Naturally-occurring mutant allele, elF4E1.S, co-dominant marker

Posted Date: January 22nd, 2020

DOl: https://doi.org/10.21203/rs.2.21512/v1

License: (c) (1) This work is licensed under a Creative Commons Attribution 4.0 International License.

Read Full License 
Version of Record: A version of this preprint was published at Euphytica on June 11th, 2021. See the published version at https://doi.org/10.1007/s10681-021-02876-y. 


\section{Abstract}

Background: The Potato Virus Y (PVY) resistance tobacco mutant VAM, which was produced by X-ray irradiation, and its derived mutant va were previously shown to contain large chromosomal deletions. Among the genes deleted in lines containing these loci is a specific eukaryotic translation initiation factor 4E (elF4E) gene, designated elF4E1.S, whose protein product has been shown to facilitate infection by certain potyviruses, the most important of which from an agronomic perspective is PVY. Because the extent of the deletions and the exact nature of their specific breakpoints have not been precisely established for VAM or va, it has not been possible to develop co-dominant markers for these loci.

Results: Here, we identified a PVY-resistant tobacco landrace Fuquanliuye with a deletion in the 3' region of the eIF4E1.Sgene. The approximate position of the 5 ' deletion breakpoint was defined by segmented amplification, cloning and sequencing. Using information from this analysis, a chromosome walk was initiated and the nucleotide sequence of the deletion junction was obtained. Through comparison with the tobacco reference genome, it was found that an approximately $27 \mathrm{~kb}$ sequence including the 3 ' region of the eIF4E1.S gene was deleted from the Fuquanliuye genome and nine extra nucleotides of unknown origin were inserted at the breakpoint. Based on the information above, a PCR-based co-dominant molecular marker for the identification of the elF4E1.S mutant allele was developed. Using this codominant marker, we genotyped F2 plants segregating for the elF4E1.S mutant allele and confirmed good consistency between the genotype and the phenotype to PVY virus.

Conclusions: We report a novel naturally-occurring mutant allele of elF4E1.Sfrom the PVY-resistant tobacco landrace Fuquanliuye, designated eIF4E1.Fu, and a PCR-based co-dominant marker specific for elF4E1.Fu. This may provide a valuable genetic resource and a basis for marker-assisted selection to improve the PVY resistance of breeding cultivars/lines.

\section{Background}

Tobacco (Nicotiana tabacum L.) is one of the most important commercial and industrial crops in the world. Potato virus $\mathrm{Y}$ (PVY) is one of the most economically important pathogens affecting tobacco worldwide, causing severe reductions in yield and leaf quality every year [1]. Selective breeding and planting of resistant varieties is the most economical, safe and effective way to control the disease. Resistance sources to PVY in tobacco can be divided into two categories: (1) TI1406 and its derivatives, with PVY resistance controlled by the recessive loci VAM or va [2, 3]; and (2) dominant resistance genes derived from Nicotiana africana that confer immunity to PVY [4]. From a commercial perspective, the va locus is of particular importance as it has been widely used in breeding for the development of PVY resistant in tobacco varieties. In order to improve breeding efficiency, researchers have developed molecular markers associated with va, including Randomly Amplified Polymorphic DNA (RAPD) and Sequence Characterized Amplified Region (SCAR) markers [5, 6, 7]. Since the genetic distances between these markers and the va locus are long, however, their utility in practical breeding applications is limited. 
A survey by Noguchi et al. [5] of 18 PVY resistant tobacco varieties using RAPD markers suggested that in each case the resistance phenotype was associated with a deletion in the same chromosomal region as $v a$, and that the sizes of the deletions varied considerably among the genotypes. Julio et al. [8] made a comparative analysis of transcripts from PVY resistant and susceptible near-isogenic lines (NILs) of tobacco using next-generation sequencing, and concluded that elF4E1.S was the tobacco gene responsible for susceptibility to PVY infection in wild type plants. Liu et al. [9] developed a dominant marker closely linked to the wild-type eIF4E1.S allele based on the sequence information of the elF4E1.S gene in tobacco, but this marker cannot distinguish heterozygotes (elF4E1.S/eif4e1.s) from both homozygous classes, which restricts its practical application in the improvement of tobacco resistance to PVY using molecular marker-assisted selection. Dluge et al. [10] made a comparative analysis of transcripts from tobacco genotypes TI 1406 (VAM), K326-va and K326 (wild type) using next-generation sequencing. It was discovered that TI 1406 and K326-va contain large deletions in the region of chromosome 21 where elF4E1.S is located. Although this study described the nature and extent of the VAM and va chromosomal deletions in considerable detail, the authors ultimately failed to obtain sequence information across a breakpoint, and thus could not develop a codominant marker that could be used by breeders for the selection of the VAM or va mutations.

The present study describes the characterization of elF4E1.S in Fuquanliuye, a PVY-resistant sun-cured tobacco landrace from Guizhou Province, China that was identified from a large scale screen of tobacco germplasm for resistance to PVY. A genomics-based analysis of Fuquanliuye in the region of elF4E1.S resulted in the precise characterization of an $\sim 27 \mathrm{~kb}$ fragment of the chromosome that was missing in this line. Using this information, we developed a co-dominant marker for the elF4E1.S gene and its mutant counterpart by designing a set of three allele-specific primers and a corresponding multiplex PCR protocol. The applicability and reliability of the co-dominant marker system was determined by evaluating the degree of concordance between the phenotype and marker genotype in an F2 population of the cross K326 $\times$ Fuquanliuye.

\section{Results}

\section{Tobacco line Fuquanliuye possesses a partial copy of elF4E1.S}

From a recent screen for PVY resistance from over 900 tobacco germplasm accessions curated by the Guizhou Academy of Tobacco Sciences, nine lines collected in China were identified as resistant (data not shown). As an initial characterization, the nine PVY resistant tobacco accessions were screened for the presence or absence of elF4E1.S using a combination of whole genome resequencing and PCR. Among the PVY resistant lines surveyed, a variety known as Fuquanliuye was selected for in depth analysis. To define the nature of eIF4E1.S within the Fuquanliuye genome, six different sets of primers spanning various portions of the gene were designed used for PCR analysis of both wild type K326 and mutant Fuquanliuye tobacco genotypes. Because PCR products were generated in both backgrounds using all six primer pairs, they were subsequently cloned, sequenced and aligned to the reference genome sequence of Nicotiana tabacum (Hongda). The sequencing results showed that the PCR products 
amplified from K326 with all primer sets were identical to the corresponding regions of elF4E1.S, and the PCR products amplified from Fuquanliuye with the primer sets $A, B$ and $C$ were also identical to the corresponding regions of elF4E1.S. Unexpectedly, however, all PCR products amplified from Fuquanliuye with the primer sets covering regions $D, E$ and $F$ were completely different from the corresponding regions of elF4E1.S (Fig. 1), and thus appeared to be products of nonspecific amplification. These results suggested that a portion of the eIF4E1.S gene in Fuquanliuye tobacco was deleted, and that the $5^{\prime}$ breakpoint of the deletion was located somewhere between bases 2261 and 2854 (Fig. 1).

\section{Cloning and verification of a junction fragment in Fuquanliyue that lacks a portion of elF4E1.S}

To genome walk across the chromosomal region in Fuquanliyue possessing a partial deletion of elF41.S, three rounds of nested-PCR were carried out as described in Materials and Methods. By the third round, a fragment of about $800 \mathrm{bp}$ began to predominate when genomic DNA of Fuquanliuye was used as the template (Fig. 2). Sequence analysis of the cloned fragment revealed that the initial $355 \mathrm{bp}$ from the $5^{\prime}$ end of this fragment aligned perfectly with the genomic DNA sequence of elF4E1.S. Thus, it could be preliminarily concluded that this amplified fragment was the desired target fragment. An antisense primer designated SP4 was designed against sequences located in the unique 3 ' end of the amplified fragment. When SP4 was coupled with the sense primer SP1, PCR reactions with genomic DNA from Fuquanliuye yielded the predicted fragment of $1051 \mathrm{bp}$ (Fig. 2). The subsequent cloning and sequencing of the 1051 bp confirmed that it matched that of the fragment obtained through genome walking. The DNA sequence of the 1051 bp fragment is shown in Fig. 3a.

Alignment of the $1051 \mathrm{bp}$ fragment unique to the Fuquanliuye genome with the current version of the China Tobacco Genome Database revealed that the sequences opposite the deletion junction in Fuquanliuye are located approximately $27 \mathrm{~kb}$ downstream in the wild type genome. Close examination of the Fuquanliuye-specific fragment and the reference genome showed that 26965 nucleotides were deleted from the Fuquanliuye genome and the nine nucleotides TTGAAAATT were inserted at the breakpoint (Fig. 3b). The deletion event in Fuquanliuye resulted in the removal of exons 2 through 5 of elF4E1.S (Fig. 3b), thus assuring its nonfunction. The novel large deletion mutation in Fuquanliuye that includes the majority of elF4E1.S was designated elF4E1.Fu. Analysis of the sequences within the deletion fragment revealed three large reading frames; one predicted to encode a protein greater than 400 amino acids in length and the other two predicted to encode proteins over 1000 amino acids in size (Additional file 1: Figure S1). In each case, however, homology searches in public databases revealed no insights with respect to potential function as all homologs were simply annotated as uncharacterized proteins. Nevertheless, given that the chromosomal deletion found in Fuquanluiye is much smaller than the deletions defining the VAM and va loci that span several Mb [10], utilization of elF4E1.Fu as a source of PVY resistance would be expected to be less disruptive to normal plant growth and development than either of the two traditional sources. The DNA sequence of the region of chromosomal DNA deleted in Fuquanluiye is shown in Additional file 1: Figure S1.

\section{Development of a co-dominant marker specific for the PVY resistance allele elF4E1.Fu}


In order to differentiate the null allele elF4E1.Fu from the wild-type elF4E1.S allele, we developed a PCRbased co-dominant marker based on the sequence analysis as described above. As shown in Fig. 3b, a common forward primer (FWm) was designed based on the sequence upstream of the 5 ' breakpoint, and a reverse primer $(\mathrm{Rm})$ specific to the null allele was designed based on the sequence downstream of the 3 ' breakpoint. Another reverse primer (RW) specific to the wild-type allele was designed based on sequences 3'proximal to the breakpoint, and thus absent in the Fuquanliuye genome. Using primers FWm/RW, a 763-bp fragment specific to the wild-type allele was amplified from homozygous susceptible plants (elF4E1.S/ elF4E1.S) and heterozygous plants (elF4E1.S/ elF4E1.Fu), but not from homozygous resistant plants (elF4E1.Fu/ elF4E1.Fu) (Fig. 4a). Using the FWm/Rm primer pair, a 572-bp fragment specific to the null allele was amplified from homozygous resistant plants and heterozygous plants, but not from homozygous susceptible plants (Fig. 4b). In both reactions, the annealing temperatures of the PCR primers for the two target fragments were identical, so a tri-primer multiplex PCR assay was attempted using the original concentrations of template DNA, primers and Premix Taq DNA Polymerase, with primers FWm, RW and Rm in equimolar concentration. The tri-primer multiplex PCR was carried out using genomic DNAs from homozygous resistant, homozygous susceptible and heterozygous plants as the template. As can be seen from Fig. 4c, the tri-primer multiplex PCR accurately identified each of the three genotypes.

To further verify the integrity of the assay, the PCR products resulting from the experiments shown in Fig. 4 were subjected to DNA sequence analysis. The sequencing results confirmed that the 763-bp fragment corresponds to the wild-type allele and the 572-bp fragment matched the sequence expected for the null allele.

\section{Validation of the codominant molecular marker in an F2 population segregating for PVY resistance}

A friction inoculation test was conducted to identify the degree of PVY resistance in 500 individual plants from an F2 population of the cross K326 × Fuquanliuye. Plant disease resistance/susceptibility was evaluated 20 days after inoculation. Among the 500 F2 plants inoculated, 122 resistant plants and 378 susceptible plants were identified, which is in accordance with the expected 1:3 segregation ratio for a single recessive resistance gene. The same 500 F2 plants were analyzed using the tri-primer multiplex PCR assay. A representative example of the results are presented in Fig. 5, where parent 1 (P1) is Fuquanliuye, parent 2 (P2) is K326. and plants 1-21 are segregating F2 plants. Consistent with expectations, plants $3,4,8,10,14,16$ and $\mathrm{P} 1$ were resistant to PVY and all other plants were susceptible. A $100 \%$ correlation between plants homozygous for elF4E1.Fu and displaying resistance to PVY inoculation was observed across the entire 500 plant population.

\section{Discussion}

It has been shown that the mutations rendering the tobacco gene elF4E1.S nonfunctional serve as recessive resistance genes against PVY. Thus, the wild type elF4E1.S gene can be considered as diseasesusceptibility gene for PVY and certain other potyviruses. Liu et al. [9] developed a dominant DNA marker 
closely linked to the recessive resistance gene va based on genomic DNA sequence information. Although this marker can distinguish some tobacco varieties resistant to PVY from susceptible varieties, it is not very useful for transferring elF4E1.S deletion mutations into PVY-susceptible recurrent parents because it can only identify individuals that are homozygous-recessive for the marker allele, and can't distinguish between homozygous dominant and heterozygous genotypes; thus requiring test crosses each generation that would double the time and effort needed to transfer the trait. Furthermore, we found that using those primers as markers could amplify the corresponding specific band in several PVYresistance sun-cured tobacco landraces identified from different provinces in China by our research team (unpublished results) Øpresumably because the mutations in elF4E1.S in those lines did not involve the deletion of the chromosomal fragment containing the marker sequences. Therefore, when screening tobacco germplasm resources for resistance to PVY, it is important to conduct a thorough characterization of the elF4E1.S gene in its entirety, as important sources of resistance, such as the elF4E1.Fu allele described herein may be missed.

Due to the recent advances in genome-editing technologies, another option for creating new sources of PVY resistance could be through the directed mutagenesis of elF4E1.S using these technologies. Though genome editing is undoubtedly a powerful tool, tobacco varieties developed using genome editing may be banned from commercial cultivation in some countries, as currently the European Union has ruled that genome-edited crops must be regulated the same as genetically modified organism (GMO) crops [11]. Therefore, in the current regulatory environment it could be argued that the discovery and exploitation of natural sources of PVY resistance, coupled with easy to use co-dominant markers corresponding to the cognate eIF4E1.S mutation represents the best strategy for developing new PVY resistant tobaccos varieties.

DNA sequence polymorphism analysis of tobacco elF4E1.S alleles of resistant and susceptible tobacco varieties is the basis for developing co-dominant markers. Studies show that tobacco PVY resistance can be broadly divided into two main types $囚$ those controlled by recessive loci such as VAM and va [2, 3], and those which are dominant, conferring an immune resistance to PVY as evidenced in Nicotiana africana [4]. Although the va locus has been deployed in several commercial tobacco varieties, the transfer of va to new tobacco varieties using backcross breeding is very difficult due to its recessive phenotype and the fact that all molecular markers targeting this locus that have been described to date are dominant, and thus cannot distinguish between homozygous dominant (VA/VA) and heterozygous ( $V A / v a)$ individuals in segregating populations. In addition to the lack of co-dominant markers specific for va, another potential disadvantage of this source of PVY resistance is the size of the deletion that defines this locus. A 2018 study of two PVY-resistant tobacco accessions carrying the VAM and va loci attempted to estimate the extent of the chromosomal regions that are missing at these loci. The VAM deletion was shown to span a minimum of $6.55 \mathrm{Mbp}$ containing 184 predicted genes; the va deletion was characterized as missing at least $5.5 \mathrm{Mbp}$ of chromosomal DNA encoding a minimum of 143 genes [10]. Furthermore, the authors of this study were not successful in identifying the location of a deletion breakpoint, information that would have allowed the development of co-dominant markers for these loci. Although a strong linkage drag has already been described for plants possessing the VAM mutation, 
most notably due to the observation that important trichome exudates are either negligible or completely missing in plants carrying this deletion [12,13], it is also plausible that negative attributes are also associated with the va locus, given the size of the deletion and the number of genes missing in plant carrying va.

The elF4E1.Fu source of PVY resistance described in this report promises to be a superior source for developing new PVY resistant varieties for several reasons. First, being about $27 \mathrm{Kbp}$ in size, the extent of the chromosomal DNA missing at elF4E1.Fu is vastly smaller than that associated with $\operatorname{VAM}(>6.55$ $\mathrm{Mbp})$ or $v a(>5.5 \mathrm{Mbp})$. In addition to the elimination of the majority of elF4E1.S, we were only able to identify three predicted genes within rest of the sequences deleted in eIF4E1.Fu, all annotated as uncharacterized proteins. Thus, it is very likely that tobacco plants utilizing eIF4E1.Fu as the source of PVY resistance will suffer less linkage drag than plants possessing va. Second, the co-dominant marker specific for elF4E1.Fu described here represents a powerful tool for tobacco breeders due to its ability to discriminate all three allelic combinations. Finally, as elF4E.Fu is a naturally occurring mutation, identified from a collection of sun-cured tobacco varieties in China, it has advantages over mutations that may be produced either via genome editing (regulatory concerns) or using nonspecific mutagens such as EMS that perturb the genome with a host of random mutations.

\section{Conclusions}

Here, we report the discovery of a novel naturally-occurring mutant allele of elF4E1.S from the Chinese tobacco landrace Fuquanliuye, designated elF4E1.Fu, which lacks a genomic region of approximately 27 $\mathrm{kb}$, including the 3'-end of elF4E1.S. Sequence information of the deletion junction enabled the development of a PCR-based co-dominant marker specific for elF4E1.Fu, which can distinguish elF4E1.S/elF4E1.Fu heterozygotes from both homozygous classes. Using this co-dominant marker, we genotyped F2 plants segregating for the mutant elF4E1.Fu allele and confirmed good consistency between the genotype and the phenotype to PVY virus. This study provides a new resistance source and an ideal co-dominant marker that can be applied in breeding resistance to potyviruses such as PVY.

\section{Methods}

\section{Plant materials and DNA extraction}

Three flue-cured tobacco varieties, K326, Honghuadajinyuan (Hongda) and Yunyan87, one sun-cured tobacco variety, Fuquanliuye, three F1 hybrids derived from crosses between the three flue-cured tobacco varieties (as female parents) and Fuquanliuye (as the male parent), and an F2 population derived from a cross between K326 (female) and Fuquanliuye (male) were used. K326, Hongda and Yunyan87 are the major flue-cured tobacco varieties planted in China, while Fuquanliuye is a PVY-resistant sun-cured tobacco landrace from Guizhou Province, China. All plant materials were collected and identified by one of authors (Rengang Wang) and deposited at the Guizhou Academy of Tobacco Science, Guiyang, China. 
Genomic DNAs of the varieties and their F1, F2 and BC1F1 derivatives were isolated from young leaves using the Axyprep ${ }^{\mathrm{TM}}$ Multisource Genomic DNA Miniprep Kit (Axygen Scientific, USA). DNA concentrations were determined with a NanoDrop 2000 Spectrophotometer (NanoDrop Technologies, Wilmington, DE, USA). All DNA samples were diluted to $80 \mathrm{ng} / \mu \mathrm{l}$ in distilled-deionized water and stored at $-20^{\circ} \mathrm{C}$.

\section{Plant inoculations}

A necrotic strain of PVY (PVY-NGZ) was isolated from a tobacco field in Guizhou Province, China, and maintained by the Guizhou Academy of Tobacco Sciences. Inoculations were carried out manually, using the sap of PVY-infected N. tabacum leaves as the inoculum.

\section{Identification of the deletion mutation of elF4E1.S in Fuquanliuye tobacco}

To characterize the region of the Fuquanliuye genome associated with elF4E1.S, six different sets of partial-overlapping primers (Table 1) spanning from -55-bp to +4423-bp with respect to the translational start site of elF4E1.S were designed and used for PCR amplifications in both wild type K326 and mutant Fuquanliuye tobacco genotypes. Amplification reactions were performed in a final volume of $20 \mu \mathrm{l}$ containing $1 \times$ PCR buffer ( $\mathrm{Mg}^{2+}$ plus), $1.0 \mathrm{U}$ Taq DNA polymerase, $0.2 \mathrm{mM}$ dNTPs, $0.4 \mu \mathrm{M}$ each of the forward and reverse primers, and $64 \mathrm{ng}$ of genomic DNA. PCR reactions were conducted in a C1000 Touch Thermal Cycler (Bio-rad, Hercules, CA, USA) with an initial denaturation step at $94^{\circ} \mathrm{C}$ for 5 min, followed by 30 cycles at $94^{\circ} \mathrm{C}$ for $30 \mathrm{~s}$, annealing temperature of $52-58^{\circ} \mathrm{C}$ for $30 \mathrm{~s}$ and extension $72^{\circ} \mathrm{C}$ for $1 \mathrm{~min}$, terminating with the final extension step at $72^{\circ} \mathrm{C}$ for $10 \mathrm{~min}$. The amplicons were purified using a PCR Purification Kit (Qiagen) and ligated into the pGEM-T easy vector (Promega) followed by transformation into competent E.coli DH5a cells (TIANGEN). Ten colonies were randomly selected for each PCR product and sequenced by Invitrogen (Shanghai, China). Sequence assembly and analysis were performed using DNAMAN software (Version 6.0). BLAST alignments were carried out using the China Tobacco Genome Database (Version 4.0).

\section{Cloning and verification of the deletion junction in Fuquanliuye}

To obtain the junction fragment originating in the 3 ' region of elF4E1.S in Fuquanliuye tobacco, a genome walking approach was initiated using the Genome Walking Kit (TaKaRa, Japan). The junction fragment was amplified by three rounds of thermal asymmetric interlaced PCR (TAIL PCR) using genomic DNA from Fuquanliuye tobacco as the template. Specific primers SP1, SP2, and SP3 (Table 1) were designed for the first, second, and third rounds of genome walking, respectively, corresponding to sequences at the 5 '-end of elF4E1.S located close to the breakpoint. The amplification reactions were performed in $50 \mu \mathrm{l}$ reaction volumes according to the manufacturers protocol, using the specific primers mentioned above and arbitrary degenerate primers provided by the Genome Walking Kit. The amplification products were cloned into the PGEM-T easy vector and sequenced. 
The genomic sequence obtained by genome walking was assembled using DNAMAN software, and the specific primer SP4 was designed from the downstream region where the sequence diverged from the wild type genomic DNA. PCR reactions utilizing SP4 together with SP1 were used in PCR reactions to validate the existence of this genomic configuration in Fuquanliuye. The PCR conditions involved an initial denaturation at 94 for $3 \mathrm{~min}$, followed by 30 cycles of $94^{\circ} \mathrm{C}$ for $30 \mathrm{~s}, 55^{\circ} \mathrm{C}$ for $30 \mathrm{~s}$, and $72^{\circ} \mathrm{C}$ for 1 min with a final extension at $72^{\circ} \mathrm{C}$ for $10 \mathrm{~min}$. PCR products of expected size was cloned into the pGEM-T vector and sequenced. The resulting fragment was compared with the reference genome of Nicotiana tabacum using the BLAST program in the China Tobacco Genome Database (Version 4.0) to identify and characterize the extent of the deletion originating from the elF4E1.S gene region in the Fuquanliuye genome.

\section{Designing and testing of allele-specific primers}

The common forward primer (Table 1; FWm) was designed according to a sequence $5^{\prime}$ of the deletion region, namely, a portion of the elF4E1.S gene that is still present in Fuquanliuye. One reverse primer (Table 1; Rm) spans the deletion region to enable amplification of the mutant eiF4E1.Fu allele, generating a PCR product of $572 \mathrm{bp}$; the other reverse primer (Table 1; RW) was designed against a sequence that had been deleted, amplifying a 763 bp product in wild type tobaccos when used with FWm. The amplification reactions were performed in a final volume of $20 \mu$ containing $1 \times$ PCR buffer $\left(\mathrm{Mg}^{2+}\right.$ plus), $1.0 \mathrm{U}$ Taq DNA polymerase, $0.2 \mathrm{mM}$ dNTPs, $0.4 \mu \mathrm{M}$ each of the forward and reverse primers, and $64 \mathrm{ng}$ of genomic DNA. The PCR cycler conditions included an initial denaturation step at $94^{\circ} \mathrm{C}$ for 5 min, followed by 30 cycles at $94^{\circ} \mathrm{C}$ for $30 \mathrm{~s}, 58^{\circ} \mathrm{C}$ for $30 \mathrm{~s}$ and $72^{\circ} \mathrm{C}$ for $1 \mathrm{~min}$, with a final extension step at $72^{\circ} \mathrm{C}$ for 10 min. The PCR products were cloned into the PGEM-T vector and and validated by DNA sequence analysis.

\section{Tri-primer multiplex PCR assay}

To simplify the PCR protocol for use in marker-assisted selection, a tri-primer multiplex PCR assay was designed. PCR amplifications were performed in a $20 \mu$ reaction volumes containing $1 \times$ Premix Taq Version 2.0 plus dye (TaKaRa, Japan), $0.4 \mu \mathrm{M}$ each of the oligonucleotide primers (FWm/Rm/RW), and $64 \mathrm{ng}$ of template DNA. Thermocycler conditions involved an initial denaturation step at $94^{\circ} \mathrm{C}$ for $5 \mathrm{~min}$, followed by 30 cycles at $94^{\circ} \mathrm{C}$ for $30 \mathrm{~s}, 58^{\circ} \mathrm{C}$ for $30 \mathrm{~s}$ and $72^{\circ} \mathrm{C}$ for $1 \mathrm{~min}$, and a final extension step at $72^{\circ} \mathrm{C}$ for $10 \mathrm{~min}$. The PCR products were analyzed by electrophoresis using $2 \%$ agarose gels.

\section{Abbreviations}

PVY: Potato Virus Y; elF4E: eukaryotic translation initiation factor 4E; RAPD: Randomly Amplified Polymorphic DNA; SCAR: Sequence Characterized Amplified Region; NILs: near-isogenic lines; TAIL PCR: thermal asymmetric interlaced PCR; GMO: genetically modified organism.

\section{Declarations}


Not applicable.

\section{Consent for publication}

Not applicable.

\section{Availability of data and materials}

All data generated or analysed during this study are included in this published article and its supplementary information files.

\section{Competing interests}

The authors declare that they have no competing interests.

\section{Funding}

This work was financially supported by the Guizhou tobacco company project $(201901,201830,201601$ and 201701). This funding body did not play any role in the design of this study and collection, analysis, and interpretation of data and in writing the manuscript.

\section{Authors' contributions}

SFL, RGW and XLR designed the research and wrote the manuscript. JY, MJL, JSZ and ZLW performed the experiments. JZ and SZY analyzed the data. RD revised the manuscript. All authors read and approved the final manuscript.

\section{Acknowledgements}

Not applicable.

\section{References}

1. Tian YP, Liu JL, Zhang CL, Liu YY, Wang B, Li XD, Guo ZK, Valkonen JP. Genetic diversity of Potato virus $Y$ infecting tobacco crops in China. Phytopathology. 2011;101:377-87.

2. Koelle G. Genetic analyse einer Y-virus (Rippenbraune) resistenten mutante der tabaksorte Virgin A. Theor and Appl Genet. 1961;31:71-2.

3. Miller RD. Registration of 'TN 86' Burley Tobacco. Crop Sci. 1987;27:365-6.

4. Lewis RS. Transfer of resistance to potato virus Y (PVY) from Nicotiana africana to Nicotiana tabacum: possible influence of tissue culture on the rate of introgression. Theor and Appl Genet. 2005;110:678-87.

5. Noguchi S, Tajima T, Yamamoto Y, Ohno T, \& Kubo T. Deletion of a large genomic segment in tobacco varieties that are resistant to potato virus Y (PVY) Mol and Gen Genet. 1999;262:822-9. 
6. Julio E, Denoyes-Rothan B, Verrier JL, Dorlhac de Borne F. Detection of QTLs linked to leaf and smoke properties in Nicotiana tabacum based on a study of 114 recombinant inbred lines. Mol Breeding. 2006;18:69-91

7. Wang G, Liu Y, Lu XP, Li KZ. (2012) Inheritance of resistance to potato virus $Y$ in tobacco and molecular marker identification. Mol Plant Breed. 2012;10:97-103. (in Chinese with English abstract)

8. Julio E, Cotucheau J, Decorps C, Volpatti R, Candresse T, Dorlhac de Borne T. A eukaryotic translation initiation factor $4 \mathrm{e}$ (eif4e) is responsible for the " $v a$ " tobacco recessive resistance to potyviruses. Plant Mol Biol Rep. 2015;33:609-23.

9. Liu Y, Song ZB, Tong ZJ, Li YP. Molecular marker from recessive gene resistant to potato virus $Y$ of tobacco and its suitability. Acta Tabacaria Sinaca. 2015;21:76-81. (in Chinese with English abstract)

10. Dluge KL, Song ZB, Wang BW, Tyler SW, Xiao BG, Li Y, Dewey RE. Characterization of Nicotiana tabacum genotypes possessing deletion mutations that affect potyvirus resistance and the production of trichome exudates. BMC Genomics. 2018;19:484.

11. Lassoued R, Macall DM, Hesseln H, Phillips PWB, Smyth SJ. Benefits of genome-edited crops: expert opinion. Transgenic Res. 2019;28:247-56.

12. Nielsen MT, Jones GA, Collins GB (1982) Inheritance pattern for secreting and nonsecreting glandular trichomes in tobacco. Crop Sci. 1982;22:1051-3.

13. Nielsen MT, Akers CP, Jarlford VE, Wagner GJ, Berger S. Comparative ultrastructural features of secreting and non-secreting glandular trichomes of two genotypes of Nicotiana tabacum Bot Gaz. 1991;152:13-22.

\section{Table}

Table 1. Primer sequences used in this study

\begin{tabular}{lll}
\hline Primer name & Primer sequence (5'-3') & Purpose \\
\hline A_F & CAAGTACCCTTTTCCTACTAAAATCTATAACTAAG & Detection of the deletion region \\
A_R & TGTTTGACTTAGCAACAACAACAAGATAAC & \\
B_F & GGACCTGAAGAAGGAGAATTGTGGAT & \\
B_R & CGTCCCAATTTATGTAATGTTGTTTGACTTA & \\
C_F & ATTGGGACGGAGGGACTATCTTG & \\
C_R & CTAACTCAAAACGGACGACTTAAATAAAGATC & \\
D_F & GACCAGTATTTCAATGTCATTCTTGGATCT & \\
D_R & CTTAAAACAATGAAAGTCTGCTCCCAC & \\
E_F & ATATCAACCACCCAAGCAAGTTAGTTGT & \\
E_R & GGCCACACCCTTGTTATCTTGC & \\
F_F & TGATGCCATTTAACAATAATGCGC & \\
F_R & CGTGGCACGAATTCCGATTACTG & \\
SP1 & AGTTTGGTAAGAGTTGGACGGGTTAG & Chromosome walking and verification of its result \\
SP2 & GTGCATTTGTCTTCATATACCAAGCTG & \\
SP3 & CTAGATTGATGAGACTAAACGTAGCTG & \\
SP4 & CCCGAAGGTTTTCACCGACG & Marker development \\
FWm & CCCATTTGACACCATAAGTTCATACG & \\
Rm & GGAGTATGATCTAGACGAAGTATCTCTTGACTAC & \\
RW & AAACACTGTTTGCAGAATTTAAGCAAC & \\
\hline
\end{tabular}


Figures

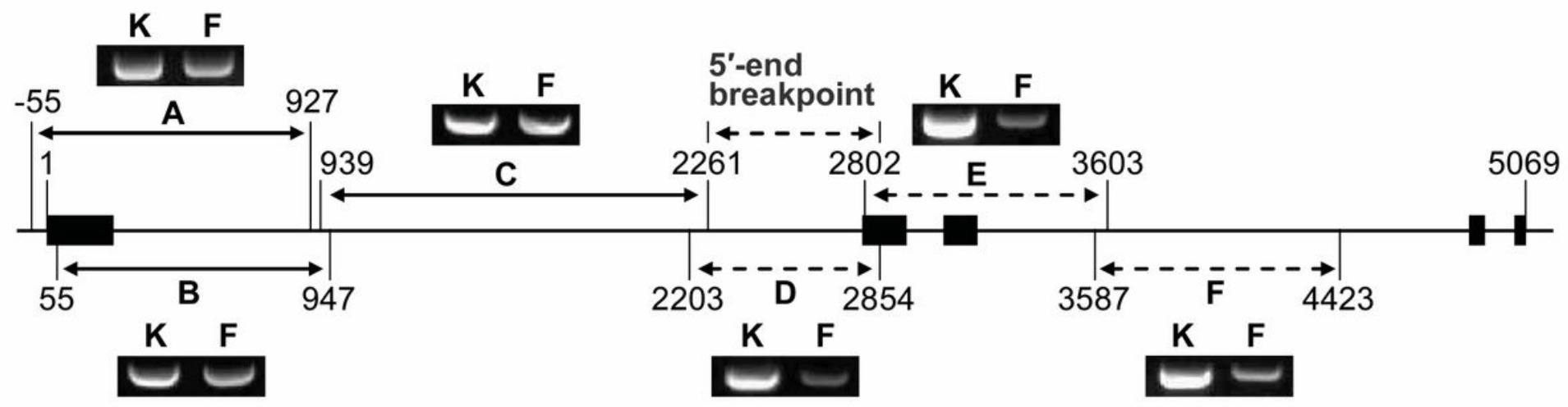

Figure 1

Breakpoint mapping of a genomic deletion of elF4E1.S in Fuquanliuye. All six primer sets yielded amplification products from wild-type and Fuquanliuye DNAs, but the 10 arbitrarily chosen clones of the PCR products of Fuquanliuye from each of primer sets $D, E$ and $F$ were non-specific sequences. One deletion breakpoint was predicted to exist between the sequences corresponding to primers $C_{-} R$ and $D \_R$ (Table 1). Exons are represented as black boxes 


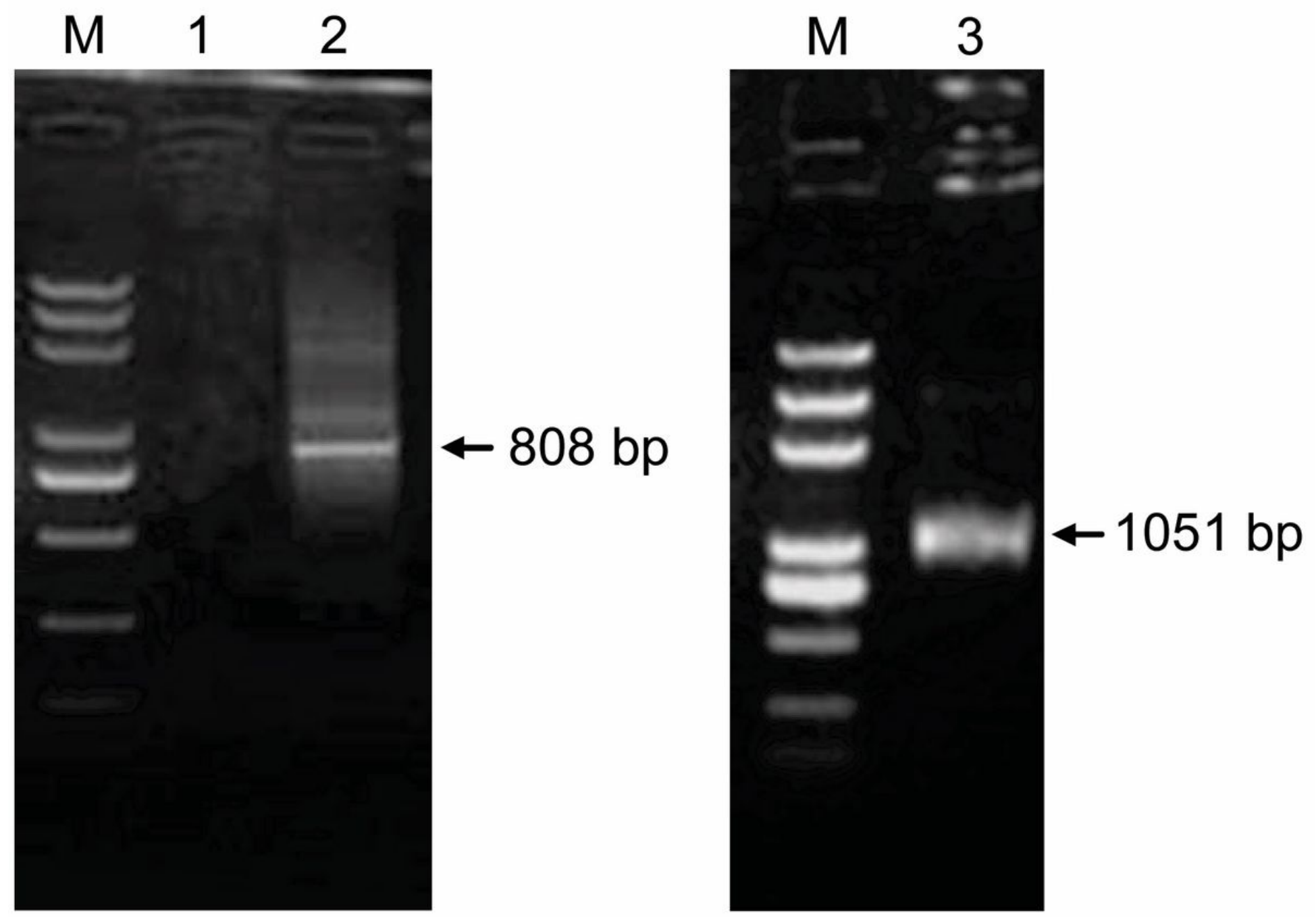

Figure 2

Agarose gel electrophoresis of amplification products of a deletion-junction fragment from Fuquanliuye. M: AL5000 DNA Marker; 1 and 2: secondary and tertiary round products of genome walking; 3: PCR products amplified from Fuquanliuye genomic DNA using primers SP1 and SP4 
(a)

5'-AGTTTGGTAAGAGTTGGACGgGTTAGGCTATGACCCAAATTTTAGCCCATCTTGACCCAACCAATC SP1

TCAGCCCAAGTAATTTTTGGGCGGATCAGTGACCCGCCCATTTGACACCATAAGTTCATACGTGTGAC GTCTCTACGTGCATTTGTCTTCATATACCAAGCTGATTATTCATTTAAAAAGATAAAGATATCTGCTTTT SP2

CTATTCCTTAATTAGGCTAAGATTACACTAATCTCCCCTCTAGATTGATGAGACTAAACGTAGCTGCA SP3

GACCAGTATTTCAATGTCATTCTTGGATCTTTATTTAAGTCGTCCGTTTTGAGTTAGGTGGTGATGATT C_R

TGAAATTTGTGTCTGCCTTAGATGCATGTTGTGTTGCTCGGATGGGGGCGCGGGTATCCCATAATGGT GCAGATCTAAAGGTCGGATTTGTCATCACATAAATTTTAGGATTCGAGGATATGAATTCAACTACGGTT ACGGGTGCTGGGATACAACCAATAAATGTATGTTACTACATATATAAGTATATATTTCGATTAATTAAAG TTATCAAACTAAATCTAATAATTTTTTCTTATAAAATATAAACACGTAATttgaaaattggaaatgaccccgacacag cagtaaatgcagatgtagtcaagagatacttcgtctagatcatactccaatggtcctgacacatttgaaaatggcgaaggtttatcccccactactccc aaacactacccaatcctctctacaaaaaaaaaaaaacattgattgaggcctgaactacgtttgacttgattccgaaaggatacgtaggcatcctctc cttgaggttcagtcacaccaacaataaaatcccattcaccctgaaattgaaaccggggcacgtcaagcggttgagaagttagcatcgtctacctttctt accaagcacaagctttcaaatcagttgccaaattggtggggaaccaataagcgtcgcaaatggagaccagcacactctgaattgagagagataaa atgagagagtctcgtcggtgaaaaccttcggg- $3^{\prime}$

\section{SP4}

(b)

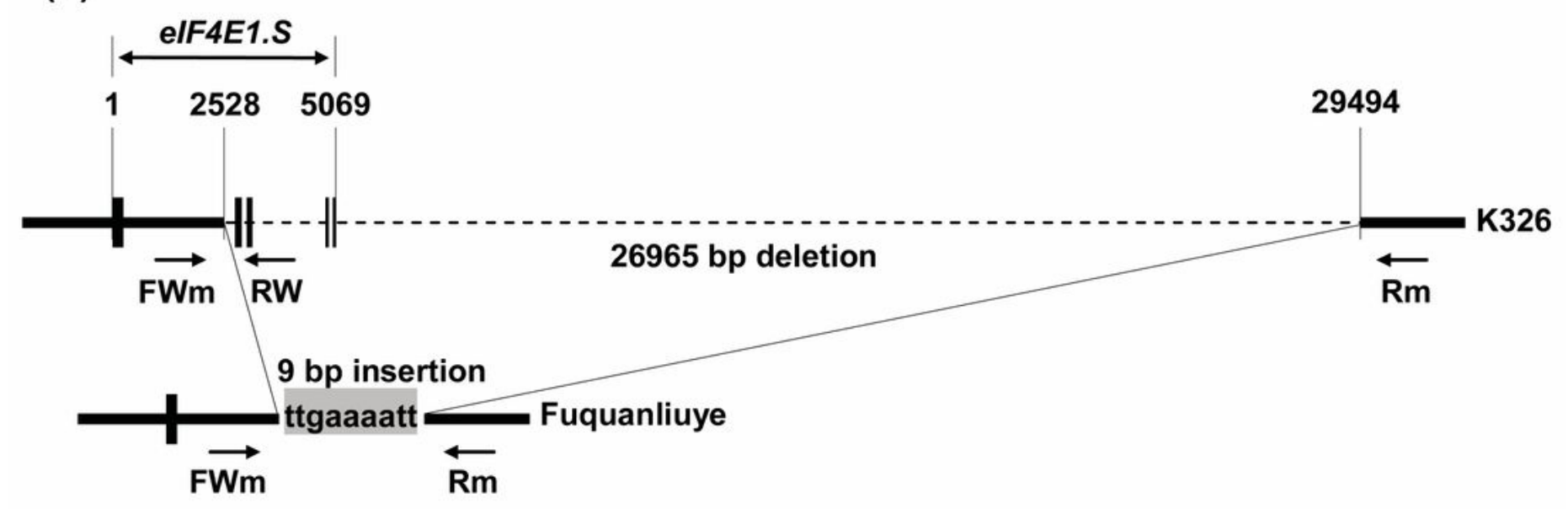

Figure 3

Nucleotide sequence and analysis of the deletion mutantion in Fuquanliuye. (a) Cloning and sequence analysis of the junction fragment of elF4E1.S gene with a 3' end deletion. Capital letters represent sequences of the residual 5' fragment of elF4E1.S in Fuquanliuye. Lowercase letters show the 3'-junction sequence obtained by genome-walking; the nine additional nucleotides inserted between the breakpoints are shaded grey. Sequences used for PCR primers are highlighted in bold and indicated by arrows. (b) 
Precise breakpoint localization of the deletion in elF4E1.S, including the positions of the three allelespecific primers designed to develop a co-dominant marker for elF4E1.S and elF4E1.Fu

(a)

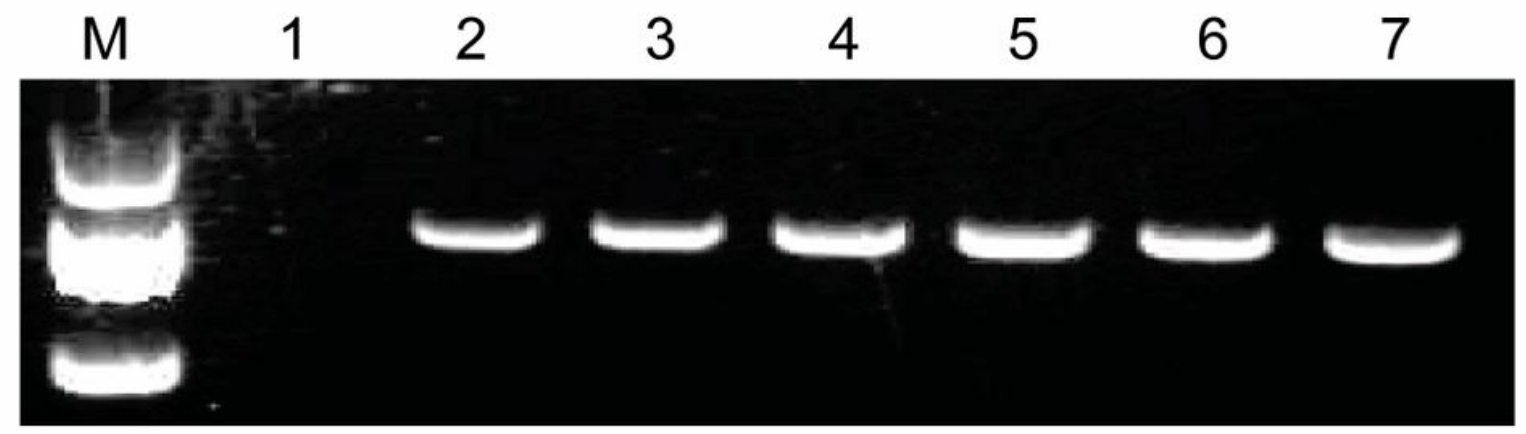

$\leftarrow 763 \mathrm{bp}$

(b)

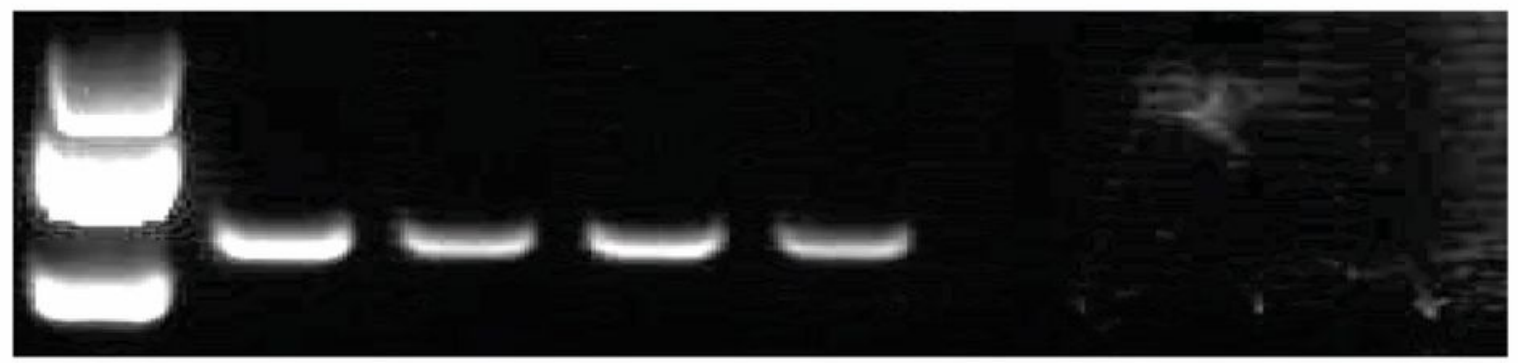

$\leftarrow 572 \mathrm{bp}$

(c)
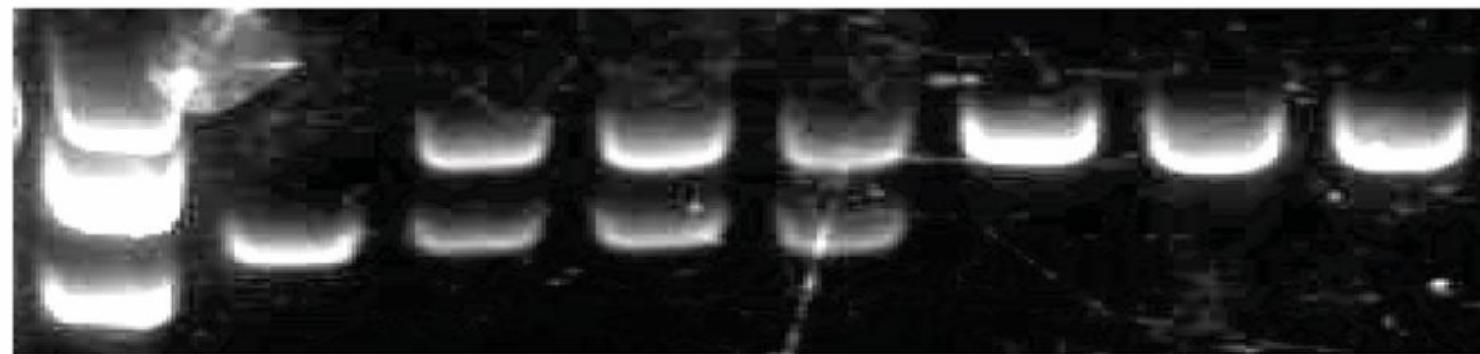

$\leftarrow 763 \mathrm{bp}$

$\leftarrow 572 \mathrm{bp}$

Figure 4

Detection of the null allele elF4E1.Fu by PCR-based markers. (a) PCR using primers FWm and RW; (b) PCR using primers FWm and Rm; (c) PCR using primers FWm , RW and Rm. M: DL2000 marker; 1:

Fuquanliuye; 2: K326 × Fuquanliuye; 3: Hongda $\times$ Fuquanliuye; 4: Yunyan87 × Fuquanliuye; 5: K326; 6: Hongda; 7: Yunyan87

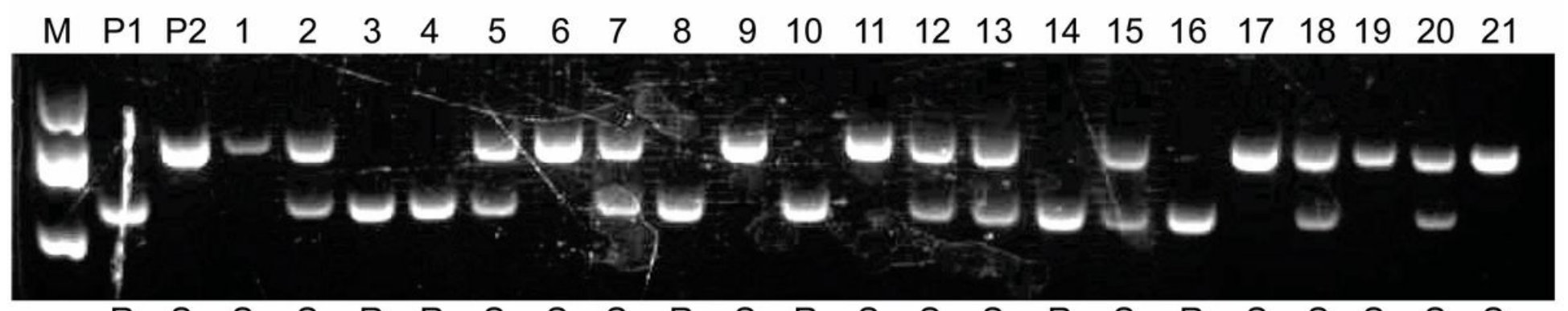

$\begin{array}{lllllllllllllllllllllll}R & S & S & S & R & R & S & S & S & R & S & R & S & S & S & R & S & R & S & S & S & S & S\end{array}$

Figure 5 
Amplification of the codominant marker in parents and representative F2 individuals from a $\mathrm{K} 326 \times$ Fuquanliuye cross. M: DL2000 marker; P1: Fuquanliuye; P2: K326; 1-21: F2 individuals. Plants 3, 4, 8, 10, 14 and 16 amplified a distinct single band of the same size as P1, plants 1, 6, 9, 11, 17, 19 and 21 amplified a distinct single band of the same size as $\mathrm{P} 2$, and the remaining individuals displayed both bands. PVY resistance phenotypes are indicated below with an R (resistant) or S (susceptible) 